\title{
LOS ORÍGENES DEL REALISMO ESTRUCTURAL: RASTREANDO LA TRADICIÓN ESTRUCTURALISTA EN FILOSOFÍA DE LA CIENCIA
}

\author{
BRUNO BORGE
}

\begin{abstract}
Current debate on Structural Realism (SR) starts when Worrall (1989) suggest this position is capable of accounting for the central intuitions of both Scientific Realism and Antirealism. Since then, it has become a rich field of debate in which complex disputes have emerged. In this paper I maintain that, (a) despite its recent appearance on the philosophical scene, SR constitutes the crowning of an epistemological trend I propose to call 'structuralist tradition'. I take as its central feature to put the focus on the relations that exist in the natural world, rather than in the nature of the entities that maintain those relations. But (b) despite this common feature, the various positions that have constituted that tradition in general, and the variants of RE in particular, have not offered a precise and uniform notion of structure.
\end{abstract}

Keywords: Structural realism; scientific realism; scientific structuralism; structuralism.

\section{Introducción}

La expresión 'Realismo Estructural' (RE) fue introducida por Maxwell (1968) para nombrar una posición alternativa a otras formas de realismo, su objetivo entonces era clarificar algunas intuiciones presentes en la obra de Schlick y (fundamentalmente) Russell. Dos décadas más tarde Worrall lleva esta posición al centro del debate contemporáneo entre realistas y antirrealistas científicos, inaugurando la última y más fértil etapa de la polémica. El RE es capaz, según Worrall, de dar cuenta satisfactoriamente de las intuiciones que sustentan los argumentos principales de ambos contendientes en esta disputa: los llamados argumento del 'no milagro' (ANM) y la meta-inducción pesimista (MIP). El primero de ellos ha servido a los realistas para justificar su creencia en la existencia de las entidades inobservables postuladas por nuestras (sucesivas) mejores teorías científicas. Según el ANM el éxito predictivo de la ciencia sería un milagro, a menos que aceptemos que nuestras teorías son verdaderas (o aproximadamente verdaderas). Los antirrealistas, por su parte, han procurado contrarrestar la plausibilidad de este argumento con la MIP: la historia de la ciencia ofrece un amplio catálogo de teorías exitosas pero finalmente refutadas, ello permite inferir inductivamente que nuestras teorías actuales se probarán, oportunamente, también falsas.

La tesis central del RE señala a los aspectos estructurales del mundo inobservable como el factor de continuidad epistémica a lo largo del cambio teórico, a lo largo de

Principia 18(2): 295-322 (2014).

Published by NEL — Epistemology and Logic Research Group, Federal University of Santa Catarina (UFSC), Brazil. 
la historia ha habido una creciente acumulación de conocimiento sobre el mundo inobservable, pero sólo acerca de su estructura. Aunque los científicos han cambiado repetidas veces de opinión acerca de la naturaleza de las entidades inobservables, su caracterización estructural ha gozado de una de una notable estabilidad. De ese modo Worrall pretende insertarse en la polémica entre realistas y antirrealistas científicos salvando lo mejor de ambos mundos. Desde entonces el RE ha motivado variadas discusiones, y ha visto nacer bajo su sombra todo un abanico de matices y posiciones. Una de las más importantes surgió a partir de que Ladyman (1998) distinguiera entre un Realismo Estructural Epistémico (REE), correspondiente a la formulación de Worrall, y un Realismo Estructural Óntico (REO), cuyo lema central es tan simple como contundente: conocemos sólo la estructura del mundo, porque es lo único que hay. Así, el REO apuesta por una reconceptualización metafísica de los objetos en términos puramente estructurales.

El RE (tanto en su versión epistémica como óntica) ha sido un tópico creciente importancia en la filosofía de la ciencia contemporánea, pero sus raíces conceptuales se hunden en las tempranas reflexiones metacientíficas del siglo XX. Los objetivos de este trabajo son (a) hacer patente el hecho de que el Realismo Estructural constituye la coronación de una tendencia epistemológica que propongo llamar 'tradición estructuralista', cuyo rasgo central ha consistido en poner el foco sobre las relaciones existentes en el mundo natural (observable y, fundamentalmente, inobservable) más que en la naturaleza de las entidades o propiedades que guardan esas relaciones, (b) mostrar que pese a ese rasgo común, las distintas posiciones que han ido constituyendo esa tradición en general, y las diversas inflexiones del RE en particular, no han ofrecido una noción uniforme y precisa de estructura. Para ello procuro describir cómo dichos rasgos se han expresado en diversas reflexiones científicas y epistemológicas a lo largo de la historia. Asimismo, en la exposición que implica la prosecución de dichos objetivos, intento conjugar tanto una presentación lógico-conceptual como una evaluación crítica de las posiciones más salientes.

En la sección 2 reviso brevemente algunas propuestas acerca de las manifestaciones históricamente más remotas de esta tradición, deteniéndome en luego en el análisis de las propuestas de Poincaré y Duhem (2.1), asimismo abordo la alternativa de Russell y la objeción presentada por Newman, que terminó por constituirse como uno de los mayores obstáculos para toda forma de REE (2.2). En la sección 3 expongo el desarrollo que las tesis estructuralistas tuvieron en el marco de posiciones ligadas al Empirismo Lógico. La sección 4 describe el tránsito hacia las formulaciones contemporáneas del RE, en tal sentido se destaca especialmente la posición de Maxwell como un puente entre dos etapas diferenciadas de la polémica; ese análisis abre el juego para la posterior exposición crítica de la propuesta de Worrall y Zahar. En la sección 5 examino dos importantes variantes de la mirada estructuralista sobre el conocimiento científico, que ejercen una fuerte influencia sobre los debates actuales: el 
Realismo Estructural Óntico (REO) de Ladyman (5.1) y el Estructuralismo Empirista de van Fraassen (5.2). Por último, la sección 6 incluye las conclusiones y comentarios finales a este trabajo.

\section{Los orígenes de la tradición estructuralista}

Las mencionadas variantes del RE se insertan en el debate contemporáneo sobre el Realismo Científico cargando el peso de un impulso histórico que las excede por mucho y que bien podríamos llamar 'tradición estructuralista'. La variedad de formas que ésta ha tomado y la debilidad con que sus primeros compases han sonado en la historia hace complejo brindar una caracterización unívoca. Pese a ello, su interés primordial en las relaciones por sobre los relata ha sido su rasgo distintivo.

John Worrall (1989) sostiene que debemos buscar los orígenes de la tradición estructuralista en la obra de Poincaré. McMullin (1990) prefiere enfatizar el rol crucial de Duhem en el trazado de los lineamientos fundantes del estructuralismo. Ambos, sin embargo, destacan a Russell como el primero en ofrecer una posición completa y articulada que sin anacronismos pueda ser cabalmente tenida por un realismo estructural. Sin dudas estos tres autores han sido antecedentes cruciales e ineludibles para el estructuralismo contemporáneo, y no hay crónica histórica que dude de ello. Sin embargo recientemente se ha procurado buscar indicios de esta tradición más allá de ellos, en lo que constituiría algo así como la prehistoria de la tradición estructuralista. Para van Fraassen (1997) el surgimiento de las geometrías no euclidianas fue un indicio de un cambio de actitud respecto de las representaciones del mundo físico, a partir del que se comenzó a admitir que no existía una manera privilegiada de representar el espacio y el mundo físico en general. En un trabajo más reciente, van Fraassen (2006) ha defendido la idea de que los antecedentes del realismo estructural pueden encontrarse ya en el siglo XVII, en el rumbo que ha marcado la teoría newtoniana. Afirma que el hecho de que dicha teoría haya terminado por adoptar descripciones sumamente abstractas y matemáticamente complejas del mundo físico ilustra el impulso de una tendencia 'estructuralista', como opuesta a una de 'reificación'. Mientras que esta última tiende a una interpretación ingenua y más bien literal de lo que en una teoría se propone, a medida que el andamiaje teórico se va complejizado y alejando de las representaciones intuitivas, se comienza a intentar capturar la realidad en sus aspectos más abstractos o matematizables.

Con todo, el interés arqueológico por encontrar actitudes o conceptos protoestructuralistas no puede soslayar el hecho de que las primeras posiciones que encarnan de modo patente tendencias estructurales no aparecen sino hasta comienzos del siglo XX. Veamos, por tanto, sus exponentes más salientes. 


\subsection{Las relaciones como una ventana a lo incognoscible}

Toda una tradición en el comentario filosófico ha hecho que el nombre de Poincaré sea asociado casi de manera automática al convencionalismo. Con todo, una nueva línea de interpretación, en la que se encuentran Worrall (1989; 1994), Zahar (1996; 2001), Psillos (1995; 1999), Gower (2000), pretende catalogarlo como el primer exponente del REE, es decir, como el primero en sostener que el conocimiento que las teorías aportan sobre la realidad se limita a las relaciones existentes entre los componentes del mundo físico (i.e. a la estructura que éstas establecen) dejando fuera toda descripción acerca de la naturaleza de esos componentes. La mencionada línea de interpretación no ha dejado de mencionar la influencia que la filosofía kantiana ejerció sobre Poincaré. Para el científico y filósofo francés, Kant era en efecto una pieza fundamental en la comprensión del conocimiento en general, y de la ciencia en particular. Pero, contrariamente a las enseñanzas de su referente, Poincaré pensaba que las cosas en sí eran al menos en cierta medida cognoscibles, ya que las identificaba con aquellas entidades no fenoménicos que son responsables de la experiencia tal como pueden ser descriptos por el discurso científico. Ahora, ¿qué tipo de conocimiento podemos esperar tener de esas entidades? Según él, "el objeto de la ciencia no son las cosas en sí mismas, como los dogmáticos en su simplicidad imaginan, sino las relaciones entre las cosas; fuera de esas relaciones no hay nada cognoscible" (Poincaré 1952, p.xxiv). Otro punto que los comentadores han coincidido en señalar es que la motivación principal para el estructuralismo de Poincaré no reside en emprender una reconstrucción conceptual o normativa de la práctica científica, sino una interpretación de sus fundamentos epistémicos que haga justicia a la historia de la ciencia. Tal como ésta caía bajo su mirada presentaba dos aspectos antagónicos, que no son más que las mismas intuiciones que en nuestros días condujeron a los argumentos del no-milagro y la MIP. Por una parte, las teorías no pueden ser simples "recetas prácticas" para hacer predicciones, dado que ello nos obligaría a atribuir su éxito al mero azar. Por otra parte, sin embargo, lo que vemos es que "las teorías duran solamente un día, y las ruinas se acumulan sobre ruinas"; pero a pesar de ello "hay algo en ellas que usualmente sobrevive. Si una de ellas nos enseña una relación verdadera, esta relación está adquirida definitivamente, y será encontrada nuevamente bajo un nuevo disfraz en las teorías que sucesivamente vengan a regir en lugar de la antigua." (Poincaré 1946, p.351). Aquello que para Poincaré garantiza la unidad del conocimiento científico a lo largo del cambio teórico es el hecho de que éste describe exitosamente las relaciones reales que las entidades inobservables mantienen. Ya en Poincaré está presente la idea de que esas realidades son capturadas por las ecuaciones matemáticas de las teorías. Esta actitud queda magistralmente ilustrada en su análisis del pasaje de la teoría ondulatoria de Fresnel a la teoría electromagnética de Maxwell. 
La teoría de Fresnel permite hacer esto [predicciones de fenómenos ópticos] tan bien como se hacía antes del tiempo de Maxwell. Las ecuaciones diferenciales son siempre verdaderas (...) ellas expresan relaciones, y si esas ecuaciones permanecen verdaderas, es porque esas relaciones conservan su realidad. Ellas nos enseñan ahora, como lo hicieron entonces, que existe tal y cual relación entre esta cosa y aquella; solamente que aquella cosa que entonces llamábamos movimiento; la llamamos ahora corriente eléctrica. Pero esos son meramente nombres para las imágenes con las que sustituimos los objetos reales que la Naturaleza esconderá por siempre a nuestros ojos. (Poincaré 1952, p.160-1)

Queda de manifiesto que si bien los cambios sucesivos de ontología son buenas razones para suponer que las teorías no nos proveen de un conocimiento acerca de la naturaleza de las entidades del mundo físico, la continuidad en las ecuaciones debe interpretarse como la adquisición acumulativa de un conocimiento acerca de las relaciones que esas entidades guardan, en otros términos, de la estructura de ese mundo. Poco hay que añadir a esas ideas para obtener formulaciones contemporáneas del REE, y así lo han visto varios defensores contemporáneos de dicha posición.

Con todo, algunas notas específicas de la noción de estructura presente en los desarrollos de Poincaré ameritan ganar mayor visibilidad, no para relativizar el rol de esta filosofía como antecedente del RE, sino para dotar al análisis histórico de una mayor profundidad. Me detendré en dos aspectos centrales. En primer lugar, el rol de la estructura matemática que pretende tener modelos en el mundo inobservable ha recibido un énfasis tal vez excesivo en la reconstrucción de Worrall, que acabó por sugerir al menos implícitamente que el conocimiento estructural preservado se agota en las relaciones entre inobservables. Esa sugerencia no es en sí misma errada si se la interpreta adecuadamente: todo cuanto subyace a la realidad fenoménica es inobservable, aquellas cosas en sí que escapan al plano de la experiencia dejan en nuestros desarrollos científicos la huella de un conocimiento objetivo estructural, pero en ese sentido particular los átomos no son diferentes de las sillas o las piedras. En otras palabras, es nuestro conocimiento de la realidad física en general el que tiene un carácter estructural, lo que pone distancia entre el RE de Poincaré y las formulaciones que siguieron a Worrall. Como veremos luego, éstas restringen el estructuralismo a las entidades inobservables mientras que mantienen un realismo del sentido común (o realismo directo) respecto de las observables. Este matiz kantiano se relaciona íntimamente con el segundo aspecto en el que considero importante poner el acento, no sólo por su naturaleza, sino por tener como resultado un distanciamiento relativo de la propuesta de Poicaré respecto de las diversas formulaciones actuales del RE. Mucho se ha insistido en la relación entre el andamiaje matemático de las teorías y la naturaleza estructural del conocimiento científico, así como en que "la única realidad objetiva consiste en las relaciones entre las cosas" (Poincaré 1952, p.352), pero

Principia 18(3): 295-322 (2014). 
se soslayado el hecho de dichas relaciones - los constituyentes de las estructuras que Poincaré invoca- son reales en un sentido muy estrecho, que a todas luces es diferente del que asumen las posiciones contemporáneas: "[S]in dudas esas relaciones (...) no pueden ser pensadas más allá de la mente que las concibe", es por ello que si se les adscribe un carácter objetivo es sólo en tanto "ellas son, van a resultar, o continuarán siendo, comunes a todos los seres pensantes" (Poincaré 1952, p.352). Las estructuras descriptas por las teorías no son aquellas presentes en la realidad en sí misma, no reflejan la estructura del mundo independiente de las mentes que las conciben, por el contrario caen del lado de esas mismas mentes, como determinaciones para todo conocimiento posible que ellas puedan formular. Como ya he señalado, estas observaciones no pretenden cuestionar el rol de Poincaré como un antecedente ineludible del RE, sino simplemente señalar que la noción de estructura que subyace a su formulación no puede identificarse sin más con la concepción que comparten las variantes del REE en la actualidad.

Al igual que Poincaré, Duhem ha sido fuertemente y por muchos motivos asociado al convencionalismo, su proximidad teórica con las tesis holistas posteriormente formuladas por Quine fue uno de ellos. Worrall (1989), Chakravarrty (1998), Gower (2000), y Zahar (2001) lo proponen sin embargo como un precursor del RE, pese a que casi unánimemente reconocen que los pasajes en los que esa tendencia se manifiesta deben ser seleccionados entre muchos otros que lo filiarían claramente al instrumentalismo. Una distinción capital en la obra de Duhem ha señalado que toda teoría tiene una parte explicativa y otra representativa. Según él, la parte explicativa es aquella que pretende capturar la realidad que subyace a los fenómenos, es decir, el mundo inobservable; mientras que la representativa se ocupa de clasificar las leyes que describen regularidades. Estas dos partes guardan una relación de dependencia, ya que la explicativa es parasitaria respecto de la representativa: todo el éxito empírico de una teoría se funda en su aspecto representativo, del cual el explicativo depende. La cuestión reside entonces en cuál es el rol epistémico que esta parte representativa tiene. Si se tratase de meras herramientas de cálculo para la derivación de predicciones, la posición de Duhem sería claramente instrumentalista. Si se tratase de un conjunto de descripciones verdaderas del mundo fenoménico (es decir, de consecuencias observacionales exitosas) que articulan con un aparato explicativo que lo trasciende, pero con el cual el científico no tiene por qué comprometerse, bien podría pensarse que esta posición anticipa el estructuralismo empirista de van Fraassen. Si en cambio se pensase que la faz representativa de las teorías refleja las relaciones entre entidades cuya naturaleza permanece oculta, entonces las pretensiones de acercar a Duhem a la tradición estructuralista son fundadas. El siguiente pasaje sugiere esa posibilidad:

estamos convencidos de que ellas [las relaciones empíricas postuladas por

Principia 18(3): 295-322 (2014). 
las teorías] corresponden a relaciones similares entre las sustancias mismas, cuya naturaleza permanece profundamente escondida pero cuya realidad no parece dudosa. (Duhem 1991, p.26)

Esa apelación a 'naturalezas escondidas' es coherente con ciertos pasajes en los que se entrevén matices realistas. Un caso interesante es su afirmación de que "un físico no puede creer (...) que un sistema capaz de ordenar tan simple y fácilmente un un vasto número de leyes, tan dispares en una primera mirada, sea un sistema puramente artificial". Es decir, que es imposible para un físico creer que el éxito de las teorías físicas es "una maravillosa proeza de azar". La creencia que juzga más adecuada en ese contexto es que las teorías representan "las relaciones reales entre las realidades invisibles" (Duhem 1991, p.28).

Pese a ello, el rol de Duhem en la tradición estructuralista se ve desdibujado, en primer término, por el hecho ya anticipado de que interpretaciones rivales encuentran apoyo similar en otras partes de su obra. En segundo lugar, porque incluso ignorando ese punto, la idea de relaciones entre sustancias ocultas o entre realidades invisibles no encuentra una formulación lo suficientemente clara y completa como para cristalizar en una noción definida de estructura.

\subsection{Estructura abstracta y conocimiento inferencial}

Ha sido Russell el primero en ofrecer una posición completa y articulada que merezca el nombre de RE, en este caso en su versión epistémica. Los primeros trazos de esa posición pueden ser encontrados en su obra The Problems of Philosophy (1912), en la que desarrollando un proyecto epistémico fundacionista que toma como base a los datos sensoriales para la construcción del conocimiento, desarrolla la idea de que esos datos son en realidad causados por objetos físicos, pero afirma que respecto de esos objetos físicos sólo podemos conocer sus relaciones: "podemos conocer las propiedades de las relaciones requeridas para preservar la correspondencia con los datos sensoriales, pero lo que no podemos saber es la naturaleza de los términos entre los cuales esas relaciones ocurren" (Russell 1912, p.15-6)

En su obra The Analysis of Matter (1927) Russell redondea esta posición convirtiéndola en un capítulo de un extenso desarrollo en el que pretende brindar una reconstrucción de la ciencia en general, y de la física en particular, a partir de una teoría causal de la percepción. Nuevamente son los datos de los sentidos los únicos objetos directos de conocimiento empírico, a partir de los cuales inferencialmente podemos conocer algo acerca de aquellos factores no sensoriales que los causan. En el marco de cierta prudencia Russell deja de hablar de 'objetos físicos' para tratarlos como las 'causas externas de la percepción'. En cualquier caso, respecto de lo único que podemos arribar a un conocimiento completo, que incluya la naturaleza intrínseca de su objeto, es respecto de los perceptos, pero no de sus causas. Para delinear esta 
posición se vale de dos principios básicos. El primero de ellos ha sido posteriormente bautizado por Psillos (1999) como el Principio Helmholtz-Weyl (H-W), dice que "asumimos que diferentes perceptos tienen diferentes stimuli" (Russell 1927, p.255), es decir, que diferentes efectos (perceptos) implican diferentes causas. El segundo es el Principio de Reflexión de las Relaciones (RR), y afirma que las relaciones entre perceptos reflejan (i.e. tienen las mismas propiedades matemáticas que) las relaciones entre sus causas no perceptuales. Lo que se desprende de la operación conjunta de estos dos principios es que si bien no pueden ser conocidas las naturalezas intrínsecas del mundo físico, se puede en gran medida inferir su estructura a partir de la estructura de nuestras percepciones. Más precisamente, los principios garantizan que la estructura del mundo físico es isomórfica respecto de la estructura de nuestra percepción. Pero dado que el isomorfismo estructural es el límite para nuestro conocimiento de las relaciones que tienen lugar en el mundo físico, lo único que podemos saber es que éste tiene al menos una estructura de relaciones isomórfica respecto de la estructura de la percepción, es decir, nuestro conocimiento del mundo externo se agota simplemente en aquello que puede ser garantizado por el isomorfismo.

A fin de clarificar este punto tal vez convenga echar mano a la distinción que Readhead (2001) traza entre estructura abstracta y estructura concreta. A una estructura concreta corresponden un dominio determinado y una clase de relaciones determinadas entre los elementos de ese dominio. Es decir, en una estructura concreta tanto los elementos del dominio como las relaciones entre ellas están especificados. La noción de estructura abstracta (que es parasitaria respecto de la de estructura concreta) corresponde a la de una estructura cuyo dominio y clase de relaciones no queda especificada sino por la adjudicación de un isomorfismo respecto de una estructura concreta dada, en otras palabras, respecto de una estructura concreta podría definirse una estructura abstracta, que no es otra cosa que la clase isomórfica cuyos miembros son solamente las estructuras isomórficas a ella. Mientras que en una estructura concreta puede conocerse tanto la naturaleza específica de los elementos de su dominio, como el tipo de relaciones que ellos guardan, en una estructura abstracta el conocimiento se limita a las propiedades matemáticas de las estructuras que son isomórficas a una estructura concreta dada.

Así, la visión de Russell podría ser descripta del siguiente modo: el mundo observable instancia una estructura concreta cuyos elementos son perceptos, definida a partir de las relaciones que postulamos/descubrimos entre ellos. Por un mero proceso de abstracción a partir de la estructura concreta de nuestra percepción puede obtenerse una estructura abstracta, i.e., la clase de todas las estructuras isomórficas a ella. Ahora bien, por apelación a los principios H-W y RR podemos inferir la existencia de una estructura concreta en el mundo físico que es isomórfica a la del mundo percibido, y cuyos elementos constituyentes (los objetos de su dominio) son causalmente responsables de los elementos constituyentes de la estructura de la percepción. Hay

Principia 18(3): 295-322 (2014). 
un proceso de abstracción que va desde la estructura concreta de lo observable hasta una estructura abstracta cuyos miembros son todas las estructuras isomórficas a la primera, pero por una deducción mediada por los principios puede inferirse que hay una estructura concreta miembro de la clase definida por la abstracta que es la estructura que corresponde al conjunto de entidades físicas que son responsables de los objetos de nuestra percepción. ¿Qué es lo que conocemos de esas entidades? Que instancian la misma estructura abstracta que nuestra percepción.

La precisión conceptual de Russell al brindar su fundamentación del conocimiento estructural del mundo físico, inédita en quienes habían ensayado sugerencias similares, no implicó la ausencia de inconvenientes. Apenas un año después de la publicación de The Analysis of Matter, Newman realiza una reseña de esta obra que incluye una de las objeciones clásicas al REE, por ello conocida como la Objeción de Newman, cuya relevancia histórica la hace un suceso ineludible en cualquier relato sobre el desarrollo de la tradición estructuralista. Según Newman la pretensión de que tenemos un conocimiento puramente estructural del mundo que hace total abstracción de sus aspectos cualitativos (es decir, de la naturaleza intrínseca de esas entidades y del aspecto intensional de las relaciones que guardan) implica la trivialización de ese conocimiento. El REE diría en suma que "hay una relación $\mathrm{R}$ tal que la estructura del mundo exterior con referencia a R es W" (Newman 1928 p.144). En otras palabras, el realista estructural epistémico sabe que el mundo exterior tiene una estructura conformada por ciertas relaciones, pero su modestia epistémica le impide conocer de qué relaciones específicas se trata. Ahora bien, esa caracterización de nuestro conocimiento del mundo exterior, señala Newman, no difiere de lo que está implicado por el siguiente teorema de la lógica: "Para cualquier agregado A, puede ser encontrado un sistema de relaciones entre sus miembros que tenga asignada una estructura compatible con el número cardinal de A" (1928 p.140). Según este teorema, la mera cardinalidad implica que existen múltiples sistemas de relaciones que pueden instanciarse en ese conjunto. Si el realista estructural epistémico afirma que de la estructura de la percepción puede inferirse la estructura del mundo físico, no está diciendo nada acerca de éste (a excepción de señalar su cardinalidad) que no se siga de la pura lógica. Puesto en otros términos, si asumimos por vía de RR y H-W que el agregado de nuestras percepciones tiene la misma cardinalidad que el agregado de sus causas, afirmar que el segundo tiene una estructura isomórfica a la estructura del primero es realizar una aserción absolutamente trivial, en tanto cualquier agregado de individuos puede instanciar la estructura que queramos a condición de que sea compatible con su cardinalidad. ¿Qué es lo que se requiere, según Newman, para que nuestro conocimiento del mundo no sea trivial?: Poder determinar qué sistema de relaciones especifico instancia el mundo físico, es decir, los aspectos intensionales de estas relaciones. Pero hacer eso implica abandonar el REE. El segundo paso de la objeción entonces está dado, y ésta toma ahora la forma de un dilema: o bien el 
defensor de REE se resigna a que el conocimiento acerca del mundo sea, a excepción de aserciones sobre su cardinalidad, trivial y a priori, o abandona las restricciones estructuralistas y por lo tanto su propia posición.

Para ilustrar más cuidadosamente la objeción podemos servirnos de un ejemplo que el mismo Newman utiliza en su trabajo.

Sea 'A' una clase de objetos dada, y 'R' una relación que se mantiene entre ciertos subconjuntos de A. Sea 'B' un segundo conjunto de objetos, también provisto con una relación ' $\mathrm{S}$ ' que se mantiene entre ciertas subclases de sus miembros (...) Por ejemplo, A podría ser un conjunto aleatorio de personas, y R la relación diádica de conocerse entre sí. Un mapa de A puede ser trazado haciendo puntos en un pedazo de papel que representen a cada persona y uniendo con una línea aquellos pares de puntos que representan personas que se conocen. Tal mapa es en sí mismo un sistema, B, que tiene la misma estructura que A, la relación que se genera, S, es en este caso 'estar unido por una línea'. (1928 p.139)

A pesar de ser extensionalmente diferentes, S y $\mathrm{R}$ instancian la misma estructura abstracta. En nuestro ejemplo, conocemos tanto los aspectos intensionales de la relación como la naturaleza de los objetos incluidos en las tuplas ordenadas que la conforman. Pero esa no es la situación que el REE plantea respecto del mundo. Para convertir el ejemplo en una analogía debiera considerarse que tenemos la hoja de papel con los puntos y las líneas trazadas en ella y que sabemos también (o al menos suponemos) que esos trazos que vemos en el papel (i.e. en el conjunto B) están causados por, y se corresponden con, elementos de un conjunto A del que absolutamente nada sabemos. En ese cuadro, si admitimos (por vía de la adopción de principios independientes) que existe una relación biyectiva entre los elementos de A y B, la única información relevante que la pieza de papel nos provee es que el conjunto A tiene la misma cardinalidad que el conjunto B, es decir, que en el conjunto A hay tantos elementos como puntos en nuestra hoja de papel. El isomorfismo estructural, sin embargo, no es novedad alguna en tanto se sigue lógicamente de esa afirmación. $^{1}$

Russell brindó una noción precisa de estructura capaz de articular con sus convicciones empiristas acerca del origen del conocimiento y su teoría causal de la percepción. Como ha quedado de manifiesto, el conocimiento estructural obtenido inferencialmente no se limita a las entidades inobservables postuladas por la ciencia, en sentido estricto toda entidad es 'inobservable' y todo nuestro conocimiento de la realidad estructural, rasgos que exponen cierta continuidad respecto de las ideas de Poincaré, como así también una tensión con las versiones actuales del RE. El problema presentado por Newman mostró las limitaciones inherentes al enfoque russelliano. En una carta dirigida a Newman, Russell admite sentirse "de algún modo avergonzado por no haber reconocido [él mismo] el problema" (Russell 1968, p.176). En

Principia 18(3): 295-322 (2014). 
esa misma misiva ensaya los lineamientos de una posible respuesta (orientada a adicionar un conocimiento de las propiedades espaciotemporales de los estímulos. Sin embargo, su desarrollo efectivo nunca tuvo lugar en su obra posterior. La objeción de Newman constituye aún hoy día, como veremos, el desafío más importante para toda formulación del REE - incluso para algunas que difieren sustancialmente del relato russelliano-, y hay un relativo consenso respecto de que dicho desafío no encuentra una solución aceptable dentro de los límites de la posición.

\section{Trazos de estructuralismo: el Empirismo Lógico}

En torno a los pilares fundamentales de la tradición en la que procuro situar al RE - propuestas que fueron o bien iniciáticas o bien que retomaron el carácter sistemático del REE de Russell- puede identificarse el surgimiento de algunas posiciones que dieron nuevas pinceladas al cuadro estructuralista. Me centraré en aquellas vinculadas de modo más o menos directo con el Empirismo Lógico. La influencia de uno de sus fundadores, Moritz Schlick, sobre el pensamiento de Russell ha sido recientemente señalada por Demopulos y Friedman (1985). Es de destacar, por ejemplo, que en su obra General Theory of Knowledge (1925) sostiene al igual que Russell una diferencia entre cualidad/contenido y estructura, postulando que nuestro conocimiento acerca del mundo se restringe exclusivamente a la última. Sin embargo, a diferencia de Russell, Schlick cree que nuestro conocimiento de la estructura del mundo no es inferencial ni está anclado a cierto conocimiento intuitivo del contenido de nuestras percepciones. La clave para entender la naturaleza del conocimiento estructural tal como Schlick lo concibe se encuentra en su distinción entre conocimiento conceptual (conceptual knowledge) y saber intuitivo (intuitive acquaintance). Mientras que este último presupone un contacto directo o 'confrontación' de un objeto conocido, y sólo de él, el conocimiento conceptual implica relacionar dicho objeto con algún otro, es decir, incorporarlo en algún sistema conceptual (Schlick 1925, p.82-3). Lo central es notar que el saber intuitivo (propio, por ejemplo, de la experiencia directa con los objetos del sentido común) no comporta conocimiento en sentido estricto. Es así que incluso si tuviésemos un saber intuitivo acerca de las entidades inobservables postuladas por las teorías eso no constituiría un auténtico conocimiento. Éste implica la aplicación de conceptos que revelen la estructuración de la realidad, lo que en ciencia toma la forma de ecuaciones. Es así que "las ecuaciones de Maxwell nos revelan la 'esencia' de la electricidad, las ecuaciones de Einstein la esencia de la gravitación. Con su ayuda, somos capaces de responder todas las preguntas que puedan surgir con respecto a los objetos de la naturaleza" (Schlick 1925, p.242). Pero esta estructura de la que tenemos conocimiento conceptual no debe ser entendida como una realidad completamente independiente de la mente, como Gower observa

Principia 18(3): 295-322 (2014). 


\begin{abstract}
Algunos principios científicos deben ser impuestos por nosotros sobre la realidad, más que por la realidad sobre nosotros, si nuestra experiencia y la consecuente teorización científica han de contar como experiencia de, y teorización sobre una realidad objetiva. Hay, en otras palabras, principios que son constitutivos de los objetos de la experiencia y la teoría. (Gower 2000, p.100)
\end{abstract}

El ingrediente kantiano de la filosofía de Schlick exige una interpretación que alinea sus desarrollos con los aspectos correspondientes de la posición de Poincaré, y por lo tanto, muestra ambas propuestas como equidistantes de los presupuestos del RE contemporáneo.

La influencia de Cassirer sobre algunos de los miembros del Círculo de Viena es bien conocida, en especial su estrecha relación con Schlick. Los rastros de ese vínculo han quedado plasmados en varios puntos de continuidad en la obra de ambos, entre los que se destacan algunas intuiciones estructuralistas. De modo coherente con lo ciertas afirmaciones de Schlick, para Cassirer "los objetos de la física son en sus conexiones nómicas, no tanto 'signos de algo objetivo' como signos objetivos que satisfacen ciertas condiciones y demandas conceptuales. De ello se desprende (...) que nunca sabemos cómo son las cosas en sí mismas, sino sólo en sus relaciones mutuas" (Cassirer 1953, p.304). La dependencia de los objetos físicos respecto de las relaciones en las que se inscriben es, sin embargo, mucho más marcada en los planteos de Cassirer, y su mismo carácter de 'objetos' parece ser no mucho más que una consecuencia de su posición en un sistema determinado de relaciones. Es así que afirma que "esas 'cosas' (...) no son asumidas como existencias independientes, presentes con anterioridad a cualquier relación, sino adquieren todo su ser (...) en primer lugar en, y con, las relaciones que se predican de ellas" (Cassirer 1953, p.36).

Estos matices han motivado a French y Ladyman (2003, p.38-41) a recurrir a la obra de Cassirer para reconstruir en perspectiva histórica los antecedentes de su propia propuesta. Según ellos Cassirer no sería solamente una pieza más en el entramado de la tradición estructuralista, sino el precursor de una de las más recientes variantes del RE, el REO. Para sostener esta interpretación, apelan al hecho de que, altamente influenciado por los revolucionarios resultados que las teorías cuántica y relativista obtuvieron en su época, Cassirer explícitamente pretende refundar el modo de concebir ontológicamente al menos algunas de las entidades claves postuladas por esas teorías. Para él "no tiene sentido adscribir [a los electrones] una existencia definida y estrictamente determinada, que de todos modos, es absolutamente inaccesible para nosotros" (Cassirer 1936, p.178). Según esta idea, los electrones no deben ser concebidos como individuos sino simplemente "pueden describirse como 'puntos de intersección' de ciertas relaciones" (1936, p.180). Si bien no hay menciones explícitas a la noción de estructura en su caracterización del tipo de conocimiento del que la ciencia nos provee, pasajes como el anterior brindan sobradas razones para tener 
a Cassirer como uno de los precursores en la tarea de ofrecer una descripción puramente estructural de las entidades del mundo microfísico que trascienda el plano meramente epistemológico, postulando un carácter esencialmente estructural para su constitución ontológica. Pese a ello, nuevamente - en estrecha relación a las líneas de continuidad trazadas con el pensamiento de Poincaré y Schlick- han de tenerse en cuenta las determinaciones ontológicas y epistémicas del espíritu kantiano de la obra de Cassirer a fin de evaluar su contribución a los desarrollos posteriores del RE.

Rudolf Carnap, uno de los más notables miembros del Círculo de Viena, no puede considerarse bajo ninguna interpretación razonable como un defensor de ninguna forma de RE. De hecho, es dudoso que pueda tenerse a su filosofía como un antecedente de dicha posición, incluso en el sentido más laxo. Sin embargo sus trabajos exponen un ejemplo de cómo los compromisos estructuralistas pueden ser elevados a un rol programático de suma importancia dentro de una propuesta epistemológica. Como es bien sabido, para Carnap todos los conceptos científicos debían ser definidos explícitamente en un marco común de base fenomenalista. Si bien el sustento epistémico está dado por la experiencia privada, el carácter objetivo del conocimiento es ganado en una descripción puramente estructural que posibilite la comunicación. Vale la pena dedicar espacio a un célebre pasaje del Aufbau que ilustra este punto:

La ciencia quiere hablar acerca de lo que es objetivo, y lo que no pertenece a la estructura sino a lo material (es decir, cualquier cosa que se pueda señalar en una definición ostensiva concreta) es, en última instancia, subjetivo. Puede verse fácilmente que la física está casi por completo desubjectivizada, ya que casi todos los conceptos físicos se han transformado en conceptos puramente estructurales (...) Desde el punto de vista de la teoría de la construcción, este estado de cosas debe describirse de la siguiente manera. La serie de experiencias es diferente para cada sujeto. Si queremos lograr, a pesar de esto, un acuerdo en los nombres para las entidades que se construyen sobre la base de estas experiencias, ello no puede hacerse entonces por referencia a contenidos completamente divergentes, sino sólo a través de la descripción formal de la estructura de estas entidades. (Carnap 1928, §16)

Debe remarcarse, sin embargo, que el compromiso estructuralista no es ontológico, ni análogo al que puede encontrase en variantes del RE. Este rasgo es coherente con su distinción entre cuestiones internas y cuestiones externas a un marco lingüístico. Para Carnap (1950) la aceptación de una teoría implica la adopción de un marco lingüístico que le es propio, en el que pueden definirse sus términos descriptivos y establecerse relaciones diversas a partir de ciertas reglas. Una vez aceptado el marco cuestiones internas pueden investigarse lógica o empíricamente y responderse a partir de las herramientas que el propio marco ofrece, las cuestiones externas son, por el contrario, meras especulaciones metafísicas sin valor cognitivo. Aceptado el

Principia 18(3): 295-322 (2014). 
marco de los números naturales tiene sentido preguntar si existe un número primo máximo, se trata de una cuestión interna cuya respuesta debe buscarse mediante el razonamiento matemático, pero si en cambio nos preguntásemos por la existencia de los números en general, estaríamos aventurándonos a una cuestión externa para la que el marco lingüístico que adoptamos no puede tener respuesta. Lo mismo estaríamos haciendo si indagásemos acerca de la existencia de los átomos: ello tiene sentido como una cuestión interna, pero como problema metafísico es cognitivamente vacío.

Los trazos estructuralistas presentes en posiciones vinculadas de modo más o menos directo al Empirismo Lógico presentan rasgos semejantes a las propuestas ya abordadas en sus relaciones de continuidad con las posiciones contemporáneas. Por una parte, los compromisos estructuralistas, sean meramente metodológicos o dirigidos a la caracterización de la realidad objetiva, son suficientemente abundantes como para considerar a las filosofías consignadas en esta sección como partícipes de la tradición estructuralista que este trabajo pretende rastrear. En algunos casos — siendo el de Cassirer tal vez el más notorio- las coincidencias conceptuales permiten considerarlas como antecedentes genuinos de vertientes contemporáneas del RE. Por otra parte, sin embargo, algunas características propias de estos desarrollos exponen una notoria discontinuidad con los términos del debate actual. Las raíces kantianas de algunas de estas posiciones ponen en primer plano un aporte subjetivo a la estructuración del mundo empírico e inobservable, una faz (común a toda mente, pero al fin y al cabo) mental de la noción de estructura que está completamente ausente en el RE tal como es entendido hoy día. Además, dichas propuestas conservan el legado de una tradición que destaca el carácter estructural del conocimiento de la realidad como una totalidad, lo que se opone a la adopción de un realismo directo respecto de lo observable. En consonancia con lo que he afirmado en la sección anterior, sostengo que estos puntos no impugnan la consideración de alguna de estas posiciones como piezas de la tradición que culmina con el RE, con todo, el análisis de sus componentes estructuralistas ha de llevarse a cabo comprendiendo el contexto específico que tienen en la filosofía de cada autor.

\section{Estructura matemática y Oraciones de Ramsey}

Grover Maxwell representa un puente entre muchos de los elementos en las posiciones hasta aquí abordadas y los términos en que se conduce el debate actual, principalmente por su adopción de las Oraciones de Ramsey como medio para definir de modo preciso el conocimiento estructural aportado por las teorías. En los años sesenta Maxwell construyó su propia versión del REE fuertemente inspirada, según sus propias palabras, en la posición de Russell, pero también incorporando algunos trazos kantianos que adjudica a la herencia de Poincaré. Precisamente en clave kan-

Principia 18(3): 295-322 (2014). 
tiana sostiene que no podemos acceder al conocimiento del mundo externo, sino sólo al reino de lo fenoménico, pero a diferencia del planteo de Kant, cree que si bien no tenemos acceso directo a las cosas en sí, como si lo tenemos al mundo fenoménico, nuestro conocimiento tanto científico como del sentido común es acerca de las cosas en sí, y es un conocimiento puramente estructural (Maxwell 1968, p.155).

Tal como lo hace Russell, Maxwell señala que todo el plano físico está constituido por entidades inobservables, no en el sentido que hoy nos es habitual, sino en uno especial de acuerdo con el cual lo único que contaría como observable serían los datos sensoriales o las impresiones sensibles, todo aquello que sea su causa, sea macro como microfísico, es tenido como inobservable. Nuestro conocimiento de esta totalidad inobservable del mundo se justifica mediante su propia versión de una teoría causal de la percepción tal que "al menos cierta subclase de características de las impresiones sensibles son isomórficas con una subclase de características de los objetos físicos" (Maxwell 1968, p.156). Para garantizar este isomorfismo, Maxwell apela al principio $\mathrm{H}-\mathrm{W}$ y al hecho de que las cadenas causales que llegan desde los objetos físicos mismos hasta nuestra sensibilidad preservan sus rasgos estructurales. Admite que, aun cuando no existen razones lógicas o conceptuales que garanticen las similitudes estructurales entre los objetos del mundo y los ítems que constituyen nuestra experiencia, tanto el sentido común como teorías bien confirmadas soportan esa presunción, y que de lo contrario cualquier tipo de conocimiento del mundo se volvería llanamente imposible.

Esta restricción epistémica a los aspectos estructurales es descripta por Maxwell en términos del conocimiento de propiedades de segundo orden (o superiores) de los objetos físicos, y de la ignorancia respecto de sus propiedades de primer orden. De ese modo las propiedades de primer orden del mundo de la percepción (por ejemplo, los colores) no reflejan en cuanto a su contenido o cualidad absolutamente nada de las propiedades de primer orden del mundo físico que tienen por causa.

Tal vez el aporte más central de Maxwell consiste en su apelación a oraciones de Ramsey.

Podemos formular proposiciones que refieran a propiedades inobservables o clases de cosas inobservables cuantificando existencialmente variables de predicado y otros términos puramente lógicos, más términos cuya referencia directa sea observable. Afortunadamente cualquier teoría puede ser transformada sin pérdida significativa de su contenido en una proposición como ésta. Lo único que se requiere es reemplazar la conjunción de aserciones de la teoría por su oración de Ramsey. (1970, p.16)

Maxwell sostiene que la proposición resultante de convertir una teoría en su oración de Ramsey refiere, aunque indirectamente, a aquellos ítems que los términos teóricos prescriben. Esa referencia tiene lugar a través de términos cuya referencia 
directa son ítems de conocimiento imediato y vocabulario puramente lógico tal como variables, cuantificadores y conectivas (Maxwell 1970, p.182-3). La oración de Ramsey, entonces, no refiere a las propiedades de primer orden directamente, sino indirectamente a través de otras de orden superior, informándonos que ellas existen y diciéndonos cuáles son sus rasgos estructurales. A pesar de las múltiples coincidencias y del hecho de que Maxwell encuentra en Russell a su antecedente más inmediato y más influyente, sus posiciones respecto del REE resultan divergentes. Mientras que la oración de Ramsey preserva la estructura lógica de una teoría, la posición de Russell insiste en que es directamente la estructura del mundo inobservable lo que inferimos a partir de nuestra percepción. Podría pensarse que la estructura de la teoría refleja de algún modo la estructura del mundo, pero esa posibilidad no es adecuadamente desarrollada por Maxwell.

Los aportes estrictamente originales de Worrall al desarrollo del RE no son tan amplios como los debates que su trabajo originó. Su mayor contribución fue hacer explícito el hecho de que el RE parece ofrecer una solución que concilia al ANM y la MIP, intuición que de un modo tácito estaba ya presente en Poincaré. Pese a ello, su artículo de 1989, Structural Realism: The Best of Both Worlds?, ha reavivado la polémica en torno a las tesis fundamentales del RE al punto tal de convertirla en uno de los tópicos de debate más frecuentado en la filosofía de la ciencia de nuestros días. Una investigación como la que el lector tienen ante sus ojos sería impensada de no haber mediado el trabajo de Worrall. Como acabo de sugerir, la motivación central de Worrall es brindar una posición que pueda salvar la intuición realista que está detrás del ANM, pero haciendo justicia a la vez a la evidencia histórica que fortalece la intuición antirrealista que ha motivado al argumento de la MIP. La carrera de Worrall tras este objetivo lo lleva a recuperar el espíritu estructuralista de la obra de Poincaré. Haciendo abstracción de la influencia neokantiana de su inspirador, Worrall sostiene que la posición que acaba de bautizar como RE es capaz de salvar las intuiciones básicas de ambos argumentos. Si bien concuerda con los defensores de la MIP en que existe una evidencia incontestable en la historia de la ciencia de una discontinuidad esencial en los postulados teóricos de nuestras (sucesivas) mejores teorías científicas, esa discontinuidad se manifiesta sólo a nivel de los compromisos ontológicos con la naturaleza de las entidades postuladas por esas teorías. Sin embargo, esa discontinuidad no es tal cuando se atiende al hecho de que las ecuaciones fundamentales de las teorías (al menos en el límite) se mantienen luego del cambio. La supervivencia del andamiaje matemático fundamental de las teorías es para Worrall prueba de que, pese a la posible sustitución de la vieja por una nueva ontología, las teorías describen el entramado de relaciones existente en el mundo inobservable. Los resultados de la MIP no son cuestionados, en tanto y en cuanto la falsedad de las teorías previas sea suficiente para concluir la falsedad de las actuales. Sin embargo, esa falsedad no implica la absoluta discontinuidad del conocimiento científico, pues 
este va describiendo de modo crecientemente más preciso la estructura oculta del mundo inobservable. Pero la discontinuidad ontológica de nuestras mejores teorías científicas, no implica que su éxito empírico tenga que quedar inexplicado o ser adjudicado a causas milagrosas, es precisamente la continuidad en las descripciones estructurales que las teorías brindan acerca del mundo lo que garantiza su adecuación empírica. Dos puntos centrales han de tenerse en cuenta. En primer término, el conocimiento estructural aportado por las teorías está dado por las ecuaciones matemáticas que son retenidas a lo largo del cambio teórico. Segundo, el compromiso estructuralista se restringe sólo al ámbito inobservable. Fuera de él, nuestro conocimiento es mejor caracterizado por un realismo directo o realismo de sentido común (dicho rasgo es común a la mayoría de las variantes actuales del realismo científico, e incluso a algunas formas de antirrealismo, como el Empirismo Constructivo de van Fraassen).

Worrall describe así su posición: el realista estructural "insiste en que es un error pensar que podemos 'entender' la naturaleza de los componentes básicos del universo" (Worrall 1989, p.122) En cambio, lo que al igual que Newton podemos descubrir son "relaciones entre los fenómenos expresadas en las ecuaciones matemáticas de las teorías, cuyos términos teóricos deber ser interpretados como genuinos primitivos" (Worrall 1989, p.122). Siguiendo a Poincaré, Worrall toma al caso Fresnel-Maxwell como parte de la evidencia histórica a favor del REE, señalando que la estructura matemática de las ecuaciones se mantiene, al menos en el límite, luego del cambio a la teoría de Maxwell. Ello atestigua un desarrollo esencialmente acumulativo en términos estructurales del conocimiento científico; si bien Fresnel estuvo absolutamente equivocado acerca de la naturaleza de la luz — que la luz consiste en vibraciones que son transmitidas a través de un medio omnipresente, el éter-, estaba sin embargo probablemente en lo cierto respecto de su estructura, i.e. respecto de que los efectos ópticos dependen de alguna cosa que vibra en ángulos rectos en dirección de la propagación de la luz, tal como las ecuaciones lo requerían.

Una cuestión que surge como un evidente desafío para el RE de Worrall es si la continuidad estructural que queda patente en el caso Fresnel-Maxwell es tan sólo un ejemplo histórico afortunado o refleja adecuadamente el proceso de acumulación progresiva de conocimiento. Readhead (2001) señala que existen algunos ejemplos históricos en los cuales la continuidad estructural es difícil de postular. Sin embargo, para Readhead aun en los casos en los que la discontinuidad en las ecuaciones matemáticas sea abrupta e inapelable, puede rastrearse una cierta afinidad entre la vieja y la nueva estructura:

\footnotetext{
Estructuras cualitativamente nuevas emergen, pero hay un sentido definido en el cual nueva estructura crece naturalmente, aunque de modo discontinuo, de la vieja estructura. Para el matemático introducir una métrica en geometría, o una no-conmutatividad en álgebra son movimientos muy natu-
} 
rales. Así que miradas desde la perspectiva correcta, las nuevas estructuras parecen surgir de un modo natural, sino inevitable, de las viejas estructuras. (Redhead 2001, p.19)

Pese a ello, en mi opinión dos cuestiones deben necesariamente ser planteadas. En primer lugar, definir con precisión lo que significa que si bien dos estructuras matemáticas puedan ser discontinuas, sean a pesar de ello, afines. En segundo término, brindar indicaciones claras respecto de cómo esta suerte de afinidad matemática de dos descripciones estructurales puede garantizar la continuidad del conocimiento.

En trabajos posteriores Worrall y Zahar (2001; y Zahar 2001) han procurado precisar la noción de continuidad estructural a lo largo del cambio teórico, el resultado fue un abandono paulatino de la idea de que el conocimiento estructural queda plasmado en la estructura matemática de una teoría, en favor de la identificación de tal conocimiento con el contenido de su Oración de Ramsey. Este cambio, sin embargo, no está exento de problemas. Como Demopoulos y Friedman (1985) han objetado, inspirándose en los resultados anticipados por Newman, el REE la versión que incorpora Oraciones de Ramsey colapsa en un mero fenomenalismo salvo por la adscripción de una cardinalidad a cierta parte del mundo inobservable. ${ }^{2}$ Revisemos con más cuidado este punto. Supongamos que tenemos una cierta teoría expresada en un lenguaje formal de segundo orden y que es finitamente axiomatizable. La conjunción de todos axiomas de esa teoría será entonces una cierta oración T. Supóngase también que es posible separar todos los predicados de la teoría en dos categorías, sea en este caso teóricos y observacionales. ${ }^{3}$ Esos predicados serán nombrados como $P_{i}$, en el caso de los teóricos y $Q_{i}$, en el caso de los observacionales (reservando $P$ y $Q$ para el conjunto de todos los predicados). La teoría entonces podría ser escrita como una oración $T\left(P_{1}, \ldots, P_{m}, Q_{1}, \ldots, Q_{n}\right)$. La Oración de Ramsey en esta teoría se obtendría limpiando de ella todos los predicados no observacionales y luego cuantificando existencialmente sobre variables de segundo orden, con lo que obtendríamos: $T_{R}:=X_{1}, \ldots, X_{m}\left(X_{1}, \ldots, X_{m}, Q_{1}, \ldots, Q_{n}\right)$. Lo que esta oración dice es que existen algunas relaciones inobservables tal que $T$ vale para esas relaciones inobservables cualesquiera fueran, y para las relaciones observables $Q$. Pero en este contexto, ¿qué significa para un realista estructural epistémico decir que $T$ es verdadera o aproximadamente verdadera? En principio $T$ se aplica a un dominio de entidades que están divididas entre observables e inobservables. El hecho de que $T$ valga para el conjunto de sus predicados observables implica que $T$ es empíricamente adecuada, es decir, que tiene un modelo empírico o modelo de datos, pero, ¿qué ocurre en el mundo inobservable? Todo lo que $T_{R}$ afirma es que existen algunas relaciones que mantienen ciertas entidades para las cuales $T$ es aplicable. De ello se sigue que el mundo inobservable tiene cierta cardinalidad, a saber: la requerida para instanciar la estructura de las relaciones no especificadas representadas por las variables de se-

Principia 18(3): 295-322 (2014). 
gundo orden de $T_{R}$, pero por fuera de esta implicación respecto de la cardinalidad, decir que $T_{R}$ es verdadera es equivalente a decir que es empíricamente adecuada. Es por eso que Demopoulos y Friedman afirman que a la luz de esta objeción el REE no es muy distinto de posiciones antirrealistas como el Empirismo Constructivo.

\section{Repensando el rol de las estructuras}

Las posiciones estructuralistas hasta aquí consignadas has compartido dos supuestos fundantes. En primer lugar, que el RE es una tesis epistémica, que pone el límite de lo cognoscible en los aspectos estructurales de determinado dominio. Segundo, que ese dominio es el de las entidades inobservables. Por diferentes razones y con objetivos antagónicos, ambos supuestos han sido puestos en crisis por dos de las posiciones que han abierto importantes campos de debate dentro de la tradición estructuralista. El primero de ellos es cuestionado por el REO. El segundo, por el EE de van Fraassen. Si bien esta última no es una propuesta realista, su influencia sobre la discusión acerca de carácter estructural del conocimiento científico hace que su inclusión en estas páginas sea ineludible.

\subsection{Realismo Estructural Óntico}

La formulación de cualquier posición realista estructural ha presupuesto hasta ahora la distinción ontológica fundamental entre relaciones y relata (entidades, objetos, individuos) reconociendo la prioridad ontológica de los segundos. En el mejor de los casos, tal como se ha señalado en la sección precedente, se ha sugerido que la continuidad estructural quedaría más adecuadamente representada por medio de una semántica que tenga a las relaciones como prioritarias, pero en cualquier caso esa transformación en el orden semántico deja intacta la suposición inicial y tiene, por el contrario, la función de ilustrar de la manera más cabal posible hasta qué punto ignoramos la naturaleza intrínseca de los constituyentes fundamentales del mundo inobservable: los relata. Pero a partir de una serie de artículos cuyo hito inaugural fue What is structural realism? (1998), James Ladyman ha planteado la posibilidad de que la restricción epistémica a los aspectos estructurales del mundo inobservable no sea en realidad restricción alguna, sino que conocemos solamente su estructura, porque ella es lo único que hay. Es decir, el compromiso estructuralista no es ya epistémico sino metafísico.

Según Ladyman, el REE sucumbe ante alguna de las objeciones clásicas que se le han interpuesto, entre ellas, la objeción de Newman. Pero también ante algunas objeciones más recientes, como la planteada por Psillos (1999) respecto de la imposibilidad que el REE tiene para establecer la distinción entre contenido y estructura. En suma, el REE parecería caer en un dilema fatal. Si se limita a los aspectos puramente

Principia 18(3): 295-322 (2014). 
estructurales (atado a una noción estándar de estructura como superveniente a los individuos) reduce la supuesta continuidad estructural del conocimiento científico a afirmaciones triviales acerca de la posibilidad de establecer relaciones arbitrarias entre un conjunto de objetos desconocidos; pero si busca en cambio superar esta caracterización que trivializa el conocimiento aportado por la ciencia dando cuenta de la supervivencia de información sustancial acerca del mundo inobservable, entonces requiere una distinción precisa entre estructura y contenido de la cual carece. Esas limitaciones conceptuales se suman al hecho de que el REE deja irresuelta la cuestión de la discontinuidad ontológica a lo largo del cambio teórico.

Pero la radicalización de los compromisos estructurales no se funda exclusivamente en las limitaciones de la versión epistémica del RE, sino que tiene también su origen en una subdeterminación metafísica descripta por la filosofía de la física en relación con la individualidad de las partículas elementales. Esta motivación extra para el REO proviene de ciertas consecuencias de la física moderna, en particular de la física cuántica. En física clásica dos partículas elementales podían ser consideradas indistinguibles con respecto a todas y cada una de sus propiedades, pero pese a ello la discernibilidad, y por tanto la individualidad, quedaban salvadas si se apelaba a la consideración de sus propiedades espaciotemporales. Dicho de otro modo, pese a ser idénticas podían ser así y todo discernibles por encontrarse en lugares y/o momentos diferentes. Pero esa posibilidad queda disuelta en el marco de la teoría cuántica: dos partículas podrían compartir todas sus propiedades, incluyendo sus propiedades espaciotemporales, y aun ser numéricamente un par de partículas. Así su naturaleza metafísica queda subdeterminada, en tanto y en cuanto ellas podrían ser vistas tanto como individuos como no-individuos. Pero quebrar esa subdeterminación a favor de la consideración de las partículas como individuos implicaría la apelación a una suerte de propiedad oculta o ecceidad medieval para sostener que dichas partículas, pese a ser idénticas e indiscernibles, son individuos independientes. Una solución más modesta consistiría pasar a considerar a esas partículas ya no como objetos, sino como puntos de intersección de ciertas relaciones modales objetivas.

La posibilidad de emprender una reconceptualización metafísica de las entidades fundamentales del mundo inobservable postuladas por la ciencia física ha suscitado las más variadas críticas. Algunas de ellas cuestionan la posición en su totalidad poniendo en duda su mera inteligibilidad; otras, en cambio, reconocen su rol como una posición conceptualmente válida dentro del mapa del debate, pero cuestionan sin embargo alguno de sus aspectos constituyentes o ciertas consecuencias filosóficas que parecen desprenderse de ella. Así, se ha imputado a los realistas estructurales ónticos el carecer de una adecuada noción de estructura para dar cuenta de la refundación metafísica que pretenden emprender, como también, entre otras cosas, carecer de las herramientas semánticas necesarias para garantizar la continuidad ontológica y epistémica a lo largo del cambio teórico. ${ }^{4}$ 
En los debates actuales, la idea de prescindir de la noción de objeto ha ido perdiendo fuerza con el surgimiento de variantes no-eliminativistas del REO. En este marco, los objetos son solamente considerados dependientes de las estructuras en las que aparecen. Más allá de estas variaciones, ha de destacarse que en el contexto de los debates acerca del REO se han ofrecido nociones sumamente heterogéneas de estructura, que van desde la aplicación de la Teoría de Grupos hasta el desarrollo de metafísicas disposicionalistas. Todas esas nociones, sin dudas, difieren notablemente de aquellas presupuestas en las versiones epistémicas del RE. Con todo, el REO se muestra como un programa pujante que no sólo ha suscitado apasionantes polémicas en torno a problemas filosóficos de larga data como la modalidad, la noción de objeto, de individuo o las leyes científicas, sino que ha incorporado debates de vital importancia en la filosofía de la física, acercando así a filosofía general de la ciencia a las filosofías especiales.

\subsection{Estructuralismo Empirista}

Desde la publicación de The Scientifc Image en 1980, van Fraassen ha sido uno de los filósofos antirrealistas más influyentes, y su nombre se ha asociado de manera indisoluble al Empirismo Constructivo. En recientes trabajos, sin embargo, ha defendido una posición novel que pretende conciliar a su Empirismo con la tradición estructuralista: el EE. Sumariamente, ha defendido la idea de que pueden rastrearse continuidades estructurales a lo largo del cambio teórico, pero que ellas atañen exclusivamente a la estructura de los fenómenos, y no al plano inobservable. De ese modo, el empirista es capaz de brindar un relato consistente del creciente éxito predictivo de la ciencia sin abandonarse a explicaciones milagrosas. Dado que la adecuación empírica es el criterio de aceptación de una nueva teoría, ésta debe ser capaz de hacer las mismas predicciones y funcionar de modo adecuado en aquellas circunstancias en las que la teoría abandonada lo hacía. Es precisamente esa restricción la que garantiza que el modo en que la nueva teoría representa el mundo preserve los rasgos estructurales propios de la vieja teoría, pero esa continuidad se manifiesta sólo a nivel de la representación de los fenómenos, y no alcanza al mundo inobservable.

Su inserción en lo que he llamado la tradición estructuralista justifica con creces que el EE sea reseñado en este recorrido histórico. Sin embargo, dado su distanciamiento del RE, me limitaré a comentar brevemente dos aspectos problemáticos de su formulación.

Pese a que el autor intenta trazar una línea de continuidad entre el Empirismo Constructivo y el EE, la adopción del segundo socava uno de los fundamentos del primero. Mientras que el empirista constructivo permanecía agnóstico respecto de la existencia de las entidades inobservables y limitaba su compromiso epistémico al conocimiento del plano fenoménico, adoptaba sin medias tintas un realismo semántico, 
abogando por una interpretación literal de las teorías tanto en sus enunciados observacionales como teóricos, es decir, la caracterización de las teorías como conjuntos de enunciados a los cuales pueden adscribirse valores veritativos. Pero la adopción del EE se enmarca en un giro de la epistemología de van Fraassen hacia la concepción semántica de las teorías, es decir, hacia una caracterización de las teorías como familias de modelos, en lugar de sistemas de enunciados. ${ }^{5}$

De acuerdo a la concepción semántica de las teorías, presentar una teoría científica es, en primera instancia, presentar una familia de modelos, esto es, estructuras matemáticas ofrecidas por la representación del objeto de estudio de la teoría. Dentro de las matemáticas, objetos isomórficos no son diferentes de modo relevante; por lo que es apropiado referirse a los objetos matemáticos como "estructuras". Dado que los modelos usados en ciencia son objetos matemáticos, las descripciones teóricas de la ciencia son, por tanto, estructurales (...). Así la concepción semántica implica una posición estructuralista: la descripción que la ciencia hace de su objeto de estudio es puramente estructural. (van Fraassen 1997, p.522).

Pero la adopción de la concepción semántica implica el abandono del realismo semántico, en tanto ya no tiene sentido hablar de una 'interpretación literal' de los modelos, ni mucho menos adscribirles valores de verdad. El giro estructuralista, con las restricciones empíricas que van Fraassen le quiere imponer, termina por ubicarlo más cerca de un escepticismo instrumentalista que del agnosticismo. De hecho Contessa (2010) ha sostenido que el EE conlleva no sólo la negación del realismo semántico, sino el abandono de su agnosticismo ontológico, ya que implica el rechazo de la tesis del realismo metafísico.

Íntimamente relacionado con esta cuestión está una dificultad extra que podríamos llamar el problema de la representación. Aun sacrificado el realismo semántico, debería haber sin embargo alguna clase de conexión referencial entre la estructura matemática y la realidad fenoménica que la satisface. El mismo van Fraassen describe así el problema: “... los modelos teóricos son estructuras abstractas (...) y todas las estructuras abstractas son estructuras matemáticas" (van Fraassen 2010, p.237) Pero, "¿cómo una entidad abstracta, como lo es una estructura matemática, representa algo que no es abstracto, algo en la naturaleza?" (van Fraassen 2010, p.240)

La solución al problema de la representación requiere de la postulación de una entidad intermedia; la estructura abstracta no se relaciona de modo directo con el mundo sino con un modelo de datos que representa los fenómenos. La relación de representación entre la teoría y los fenómenos queda ahora caracterizada como un isomorfismo entre el modelo teórico y el modelo de datos que, al igual que el primero, es también una entidad abstracta. Una primera reserva podría tenerse respecto de que esto no constituya una solución sino un mero desplazamiento del problema de la representación a la relación entre el modelo de datos y los fenómenos. Si bien 
van Fraassen no ataca este problema en forma directa, se desprende de sus escritos que la relación de representación entre el modelo de datos y los fenómenos no es de isomorfía (de hecho no podría serlo en tanto los fenómenos no pueden constituir un dominio bien definido para ninguna función ${ }^{6}$ ) como en el caso de la relación entre los modelos teórico y de datos, sino de una naturaleza diferente. El modelo de datos es algo así como el output de un proceso de abstracción que tiene por inputs a los resultados brutos de los procesos de medición llevados a cabo en el marco de la teoría. Pero la inclusión de los procesos de medición como parte constitutiva de la relación de representación entre la teoría y los fenómenos, aun cuando pueda constituir una solución satisfactoria al problema de la representación, corre serios riesgos de chocar contra la pretensión de modestia ontológica del antirrealismo. Según van Fraassen, "La relación teoría/fenómenos (...) es la de una integración de una estructura matemática en otra. Dado que el modelo de datos —o más precisamente, el modelo de superficie- que representa las apariencias, es él mismo un modelo matemático." (van Fraassen 2010, p.252). Pero, "No hay nada en la estructura abstracta misma que pueda determinar que es el modelo de datos relevante para ser incorporado a la teoría" (van Fraassen 2010, p.253). Sin embargo, "un modelo de datos particular es relevante porque fue construido sobre la base de unos resultados recolectados de cierta manera, seleccionados por criterios de relevancia específicos, en ciertas ocasiones, en una configuración práctica experimental u observacional diseñada a tal propósito" (van Fraassen 2010, p.253). En otras palabras, "los fenómenos (...) no determinan qué estructuras son modelos de datos para ellos, eso depende de nuestra atención selectiva sobre los fenómenos, y de nuestras decisiones de atender a ciertos aspectos, de representarlos en ciertos modos y hasta cierto punto." (van Fraassen 2010, p.254).

No hace falta hacer notar el sesgo pragmático que adquiere la noción de representación científica construida sobre los intereses específicos que motivan los procesos de medición y selección de datos. Desde los trabajos de Hempel, y tal vez desde mucho antes, sabemos que recolectar y clasificar datos presupone ya hipótesis teóricas; eso no es un problema para la concepción semántica más de lo que lo es para la concepción sintáctica de las teorías. Lo que resulta llamativo —en mi opinión- es que nuestro modo de representar científicamente la realidad, tal como van Fraassen lo caracteriza, debe detenerse en la frontera de lo observable. Después de todo, la postulación de entidades que están más allá de las apariencias ha mostrado ser un recurso pragmáticamente relevante —y de hecho ineludible- en la práctica científica. No sólo eso: la suposición de su existencia real también lo ha sido. La restricción de los compromisos ontológicos al ámbito de la experiencia se fundaba, en el Empirismo Constructivo, en una distinción entre entidades observables e inobservables que van Fraassen se ocupó de defender con uñas y dientes, y no sin razón: después de todo hay cosas que podemos ver y cosas que no podemos ver. Pero no resulta claro cómo 
esa distinción se mantiene en los modelos de datos — pragmáticamente construidoscon la fuerza suficiente para justificar que la continuidad estructural existente en las subestructuras empíricas de nuestras (sucesivas) mejores teorías científicas es la única responsable del éxito de la ciencia, mientras que la continuidad de los modelos teóricos nada tiene que ver con la explicación de dicho éxito.

\section{Conclusión}

Pese a que su aparición formal en el escenario filosófico suele tenerse por reciente, el RE se inscribe en una extensa historia conceptual a la que - como he propuestobien le cabe el nombre de tradición estructuralista; y en tanto tal, ésta es tan antigua como los cimientos de nuestra actual filosofía de las ciencias. Más allá de los esfuerzos por intentar encontrar vestigios que configuren la prehistoria de dicha tradición, es claro que su primera manifestación cabal se encuentra en la obra de Poincaré, donde no sólo el rasgo central del estructuralismo (a saber, el poner énfasis sobre las relaciones más que en la naturaleza de las entidades o propiedades relacionadas) se hace patente, sino se manifiesta como una tesis que prescribe una limitación a nuestra penetración epistémica en el mundo natural. Indicios de un espíritu semejante pueden encontrarse en algunos pasajes de la obra de Duhem, aunque ha de reconocerse que su interpretación en clave estructuralista es quizás demasiado arriesgada. Bien distinto es el caso de Russell, quien puede tenerse por el primer filósofo en defender el REE

Esta primera formulación sistemática del REE ha dado lugar a su primera y más peligrosa amenaza: la Objeción de Newman es hasta nuestros días el reto más difícil para el REE. Las reformulaciones del REE echaron mano de oraciones de Ramsey para conducirlo paulatinamente desde una versión del empirismo clásico (tal como Russell lo proponía) hacia el terreno más restringido de los compromisos metafísicos sobre el mundo microfísico. En esa transición, la Objeción de Newman se dio por neutralizada, o fue simplemente olvidada. Sin embargo sostengo que las razones detalladas en la sección 4 son suficientes para suscribir la difundida opinión de que esa presunción era equivocada. Puede pensarse que en parte por ello se ha visto en la profundización de los compromisos estructuralistas al plano óntico la solución de algunos de los problemas crónicos del REE. Por otra parte, y pese esas dificultades, la caracterización estructural del conocimiento científico que en buena medida fue desarrollada en el marco del RE, ha influenciado fuertemente a una de las posiciones antirrealistas más sólidas, como lo es el Empirismo Constructivo de van Fraassen. En tal sentido, el REO y el EE constituyen propuestas de vital importancia para comprender el estado actual, ya no del debate en torno al Realismo Científico, sino de la filosofía de la ciencia en general.

Principia 18(3): 295-322 (2014). 
Pese a su importancia a lo largo de los rasgos de continuidad señalados, el recorrido que he propuesto expone algunas profundas tensiones $-\mathrm{y}$ en varios casos notables quiebres - en las distintas caracterizaciones de la noción de estructura. Esta discontinuidad es esperable en una tradición tan basta, donde la precisión no es ejercida de modo homogéneo en todas las presentaciones. Más allá de esa salvedad, sin embargo, se hace necesario mencionar que muchas reconstrucciones actuales de esta tradición tienden a soslayar estas diferencias. La propia reconstrucción de Worrall es un ejemplo de ello en tanto ignora los aspectos subjetivos inherentes al concepto de estructura de Poincaré, como así también el alcance específico de sus compromisos estructuralistas. Con todo, esas divergencias no se encuentran sólo en la tradición a nivel global, sino en muchas de sus expresiones particulares. Como hemos visto, la mera distinción entre REE y REO conlleva una profunda diferencia en el modo en que las estructuras son concebidas. Asimismo, la transición de las etapas más tempranas (en las que la influencia neo-kantiana era casi la regla) a las formulaciones contemporáneas del RE expone variaciones no menos importantes. Incluso al interior del REE actual pueden encontrarse tensiones en la caracterización de la noción de estructura. No es claro, por ejemplo, como la idea de que es el aparato matemático de las teorías el portador del conocimiento estructural armoniza con la apelación a Oraciones de Ramsey; además, ambos enfoques presentan serios problemas, siendo el más serio de ellos la Objeción de Newman.

Más allá de las particularidades de cada una de sus alternativas y de los elementos de discontinuidad señalados, el RE ha motivado profundos debates sobre los presupuestos filosóficos de la tradición en la que se inscribe, incorporando reflexiones metateóricas de suma importancia para brindar un marco conceptual capaz de acoger los resultados de la ciencia moderna. Sus desarrollos en ambos aspectos han sido notables. Ha logrado capturar las intuiciones básicas (incluso las más cotidianas) sobre una ciencia que si bien se equivoca muy frecuentemente parece sin embargo estar siempre en lo cierto. Esa fuerza intuitiva ha tomado la forma de una respuesta convincente a la tensión conceptual de los argumentos del no-milagro y la MIP. Eso le ha permitido consolidarse como una alternativa sólida adentro del marco del debate, gozando del privilegio de ser una posición relativamente joven, que corona sin embargo la herencia de una tradición que se remonta hasta los orígenes mismos de lo que llamamos hoy filosofía de las ciencias.

\section{Agradecimientos}

El presente trabajo ha sido realizado gracias al apoyo de la Agencia Nacional de Promoción Científica y Tecnológica (ANPCyT) y la Universidad de Buenos Aires. Algunas de sus ideas centrales fueron presentadas en el II Congreso de la Sociedad Filosófica del Uruguay, que tuvo lugar en la ciudad de Montevideo, entre el 11 y el 16 de agosto de 2014; agradezco

Principia 18(3): 295-322 (2014). 
los valiosos comentarios de la audiencia que sirvieron para mejorar la versión final de este trabajo. Por la misma razón expreso mi agradecimiento hacia los árbitros anónimos de esta revista.

\section{Referencias}

Ainsworth, P. 2009. Newman's Objection. British Journal for the Philosophy of Science 60: 135-71.

Borge, B. 2013a. Hacia una ontología sin objetos: una defensa del Realismo Estructural Óntico en el nivel metodológico. Filosofía Unisinos 14(1): 45-51.

—. 2013b. £Qué es el Realismo Estructural Óntico?: una aproximación al debate actual sobre el Realismo Científico. Revista Colombiana de Filosofía de la Ciencia 13(27): 149-75.

Carnap, R. 1928. Der logisches Atfbau der Welt. Berlin: Schlachtensee Weltkreis-Ver-lag.

- 1956. Meaning and Necessity: a study in semantics and modal logic. Chicago: University of Chicago Press, 2nd edition.

Cassirer, E. 1936. Determinism and Indeterminism in Modern Physics. Yale: University Press.

- 1953 [1910]. Substance and Function, and Einstein's Theory of Relativity (Trad. W. C. Swabey y M. C. Swabey). New York: Dover Publications.

Chakravartty, A. 1998. Semirealism. Studies in History and Philosophy of Science 29A(3): 391.

Contessa, G. 2010. Empiricist Structuralism, Metaphysical Realism, and the Bridging Problem. Analysis Reviews 70(3): 514-24.

Demopoulos, W.; Friedman, M. 1985. Critical notice: Bertrand Russell's The Analysis of Matter: Its historical context and contemporary interest. Philosophy of Science 52: 621-39.

Duhem, P. M. M. 1991[1914]. The Aim and Structure of Physical Theory. Princeton (NJ): Princeton University Press.

French, S.; Ladyman, J. 2003. Remodelling structural realism: Quantum physics and the metaphysics of structure. Synthese 136: 31-56.

Gower, B. 2000. Cassirer, Schlick and 'structural' realism: The philosophy of the exact sciences in the background to early logical empiricism. British Journal for the History of Philosophy 8: 71-106.

Ketland, J. 2004. Empirical adequacy and Ramsification. British Journal for the Philosophy of Science 55(2): 287-300.

Ladyman, J. 1998. What is structural realism? Studies in History and Philosophy of Science 29: 409-24.

Maxwell, G. 1968. Scientific methodology and the causal theory of perception. I. Lakatos; A. Musgrave (Eds.) Problems in the philosophy of science. Amsterdam: North-Holland Publishing Company.

- 1970. Structural realism and the meaning of theoretical terms. S. Winokur; M. Radner (Eds.) Analyses of theories, and methods of physics and psychology. Minneapolis: University of Minnesota Press, p.181-92.

McMullin, E. 1990. Comment: Duhem's middle way. Synthese 83: 421-30.

Newman, M. H. A. 1928. Mr. Russell's causal theory of perception. Mind 37: 137-48.

Psillos, S. 1995. Is Structural Realism the Best of Both Worlds? Dialectica 49: 15-46.

- 1999. Scientific Realism: How Science Tracks Truth. London: Routledge.

Principia 18(3): 295-322 (2014). 
Poincaré, H. 1946 [1913]. The Value of Science. Traducido por George B. Halsted. In: H. Poincaré, The Foundations of Science: Science and Hypothesis, The Value of Science, and Science and Method. Lancaster, PA: The Science Press.

- 1952 [1905]. Science and Hypothesis. New York: Dover.

Redhead, M. 2001. 'Quests of a realist', review article of Stathis Psillos's scientific realism: how science tracks truth. Metascience 10(3): 341-47.

Russell, B. 1912. The Problems of Philosophy. Oxford: Oxford University Press

- 1927. The Analysis of Matter. London: George Allen \& Unwin. 1968. The Autobiography of Bertrand Russell, vol. 2. London: Allen \& Unwin.

Schlick, M. 1925 [1918]. General Theory of Kniowledge. Traducido por A. E. Blumberg and H. Feigl. New York: Springer-Verlag.

van Fraassen, B. C. 1980. The Scientific Image. Oxford: Oxford University Press. . 1997. Structure and Perspective: Philosophical Perplexity and Paradox. In: M.L. Dalla

Chiara et al. (eds.) Logic and Scientific Methods, Dordrecht: Kluwer Academic Press.

- 2006. Structure: Its shadow and substance. The British Journal for the Philosophy of Science 57: 275-307.

- 2010. Scientific representation: Paradoxes of perspective. Analysis 70(3): 511-14.

Worrall, J. 1989. Structural Realism: The Best of Both Worlds? Dialectica 43: 99-124.

Worrall, J.; Zahar, E. 2001. Ramsification and structural realism. Appendix in E. Zahar, Poincarés Philosophy: From Conventionalism to Phenomenology. La Salle: Open Court, p.236-51.

Zahar, E. 1996. Poincaré's Structural Realism and his Logic of Discovery. In: Jean-Louis Greffe;

Gerhard Heinzmann; Kuno Lorenz (eds.) Henri Poincaré: Science and Philosophy. Berlin: Academie Verlag and Paris: Albert Blanchard.

— 2001. Poincarés Philosophy: From Conventionalism to Phenomenology. Chicago and La Salle: Open Court.

BRUNO BORGE

Departamento de Filosofía Becario Doctoral de la Agencia Nacional de Promoción Científica y Tecnológica Agencia Nacional de Promoción Científica y Tecnológica (ANPCyT) brunojborge@gmail.com

Resumo. O debate atual sobre o Realismo Estrutural (RE) se inicia quando Worrall (1989) sugere que esta posição é capaz de dar conta das intuições centrais tanto do Realismo quanto do Antirrealismo Científicos. Desde então se converteu em um rico campo de debate intelectual no qual há profundas disputas. No presente trabalho sustento que (a) apesar de sua aparição recente na cena filosófica, o RE constitui a coroação de uma tendência epistemológica que proponho chamar 'tradição estruturalista', cujo traço central consiste em colocar o foco sobre as relações existentes no mundo natural muito mais do que nas entidades que estão nestas relações; porém (b) apesar deste traço comum, as distintas posições que foram constituindo esta tradição em gera, e as variantes do RE em particular, não ofereceram uma noção uniforme e precisa de estrutura.

Palavras-chave: Realismo estrutural; realismo científico; estruturalismo científico; estruturalismo.

Principia 18(3): 295-322 (2014). 


\section{Notas}

${ }^{1}$ Un interesante recorrido histórico conceptual por el devenir de esta objeción puede encontrase en Ainsworth (2009).

${ }^{2}$ Ketland (2004) se ha encargado de ofrecer las pruebas formales que respaldan las objeciones conceptuales de Demopoulos y Friedman.

${ }^{3}$ El criterio de separabilidad se asocia frecuentemente al carácter 'problemático' o 'no problemático' de los predicados. En el contexto del programa estructuralista que abraza la concepción semántica, por ejemplo, esta distinción se realiza entre predicados T-teóricos y T-no teóricos, según sean propios de la teoría o provengan de alguna teoría previa. Claramente en el contexto del REE la división relevante debe hacer referencia a su carácter observacional o teórico.

${ }^{4}$ Para una revisión detallada de algunas de las objeciones consignadas véase Borge (2013a). Un panorama general de las variantes del REO encontrarse en Borge (2013b).

${ }^{5}$ Aunque de modo menos comprometido, su simpatía por la concepción semántica ya está presente en The Scientific Image (1980) al caracterizar la propia noción de adecuación empírica.

${ }^{6}$ Véase (van Fraassen 2010, p.241)

Principia 18(3): 295-322 (2014). 